• Entomologica Fennica. 20 September 1996

\title{
Faunistic records on Buprestidae and Cerambycidae (Coleoptera) from Greece
}

\author{
Päivö Somerma, Rauno Väisänen, Ole Mehl \& Svatopluk Bíly
}

Somerma, P., Väisänen, R., Mehl, O. \& Bíly, S. 1996: Faunistic records on Buprestidae and Cerambycidae (Coleoptera) from Greece. - Entomol. Fennica 7: 51-54.

Faunistic records are given for 17 species of Buprestidae and 40 species of Cerambycidae from Greece, mostly from the northern Pindus range, and from Mt. Pilion and the island of Skiathos.

Päivö Somerma, Laiduntie 18, FIN-02340 Espoo, Finland

Rauno Väisänen, Finnish Forest and Park Service, Vernissakatu 4, Box 94, FIN-01301 Vantaa, Finland

Ole Mehl, Birkidvej 18, Asp, DK-7600 Struer, Denmark

Svatopluk Bíly, Národni Muzeum v Praze, Kunratice c. 1, CZ-148 00 Prague 4, Czech Republic

Received 23 February 1994, accepted 13 March 1996

The buprestid and cerambycid fauna of Greece is still relatively poorly known (e.g. von Demelt 1967, 1982, Berger 1987, Markalas 1991). For example, von Demelt (1967) recorded 90 cerambycid species from Greece collected mainly from northern Peloponnesos. Later von Demelt (1982) recorded 54 cerambycid species from mainland Greece. Thus, the cerambycid fauna is relatively rich. This is apparently true also for the buprestids. It may be mentioned that at least 386 buprestid species belonging to 35 genera are known from Turkey (Lodos \& Tezcan 1990) and at least 261 cerambycid species from Italy (Sama 1988).

The authors Päivö Somerma (P.S.) and Rauno Väisänen (R.V.) made two expeditions to Greece in 1991. The principal study areas were Zagoria in the northern Pindus range and Skiathos and Mt. Pilion in eastern Magnesia. Although the main concern was on Lepidoptera, Buprestidae and Cerambycidae were also collected. The material was largely collected in the daytime by nets or on flowers and some of it by light traps. The present paper summarizes the results of those collecting trips, and supplements them with the results of previous visits in Greece. The main collecting areas are shown in the map (Fig. 1). The buprestid material was mostly identified by Svatopluk Bíly and the cerambycid material by Ole Mehl.

\section{Buprestidae, subfamily Julodinae}

Julodis pubescens (Oliver, 1790) - Peloponnesos, Olympia, 30-40 m, 9.-10. VI. 1987, P.S., 1 ind. - Zakynthos, Laganas, about $40 \mathrm{~m}$, dry meadow slope, 16. VI. 1987, P.S., abundant on grasses in the morning.

\section{Subfamily Acmaeoderinae}

Acmaeodera crinita (Spinola, 1838) - Thessaly, Skiathos, 25. VI. 1991 P.S. \& R.V., 1 ind.

Acmaeoderella flavofasciata (Piller, 1783) - Skiathos, Megaloammos, 3. VII. 1991, P.S. \& R.V., 1 ind. 


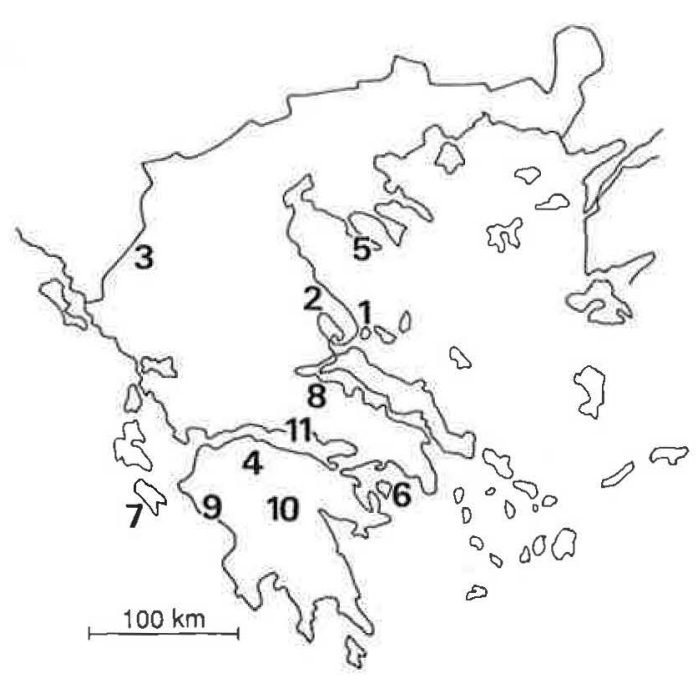

Fig. 1. Main collecting localities in Greece. 1: Skiathos; 2: Mt. Pilion; 3. Zagoria; 4: Kalavrita; 5: Kassandra, Haniotis; 6: Egina; 7: Zakynthos; 8: Kamena Vourla; 9: Olympia; 10: Vitina

\section{Subfamily Buprestinae}

Aurigena margotana Novak, 1983 - Crete, Iraklion, near the University, 19. IV. 1990, P.S. \& R.V., 3 ind.

Capnodis carbonaria Klug, 1829 - Delfoi, dry slope with some cypresses, 16. VI. 1986, P.S., 1 ind.

Buprestis dalmatina Mannerheim, 1837 - Thessaly, Skiathos, Karavotsakisma-Madraki, pine forest, 6. VII. 1991, P.S. \& R.V., abundant. — Skiathos, Koukonaris, 6. VII. 1991, P.S. \& R.V., abundant.

Poecilonota variolosa (Paykull, 1799) - Peloponnesos, Olympia, 30-40 m, river valley, 9.-10. VI. 1987, P.S., 1 ind. - Meteora, 13.6.1988, P.S., 10-20 ind. - Delfoi, 17.6.1986, P. S. about 10 ind. - All individuals on trunks of Populus species.

Trachypteris picta decastigma (Fabricius, 1787) Thessaly, Skiathos, Megaloammos, 7. VII. 1991, P.S. \& R.V., 3 ind. - Egina, Ag. Marina, 22. VI. 1986, P.S., 1 ind. - Kassandra, Haniotis, 11. VI. 1985, P. S., 1 ind. - Meteora, 13. VI. 1988, P.S., 1 ind.

Oxypteris cuspidata (Klug, 1829) - Thessaly, Skiathos, 3. VIII. 1991, P.S. \& R.V., 2 ind.
Anthaxia millefolii (Fabricius, 1801) - Thessaly, Skiathos, 25.VI. 1991, P.S. \& R.V., 3 ind. -- Egina, Ag. Marina, 22. VI. 1986, P.S., 1 ind.

Anthaxia umbellatarum (Fabricius, 1787) - Thessaly, Skiathos, 3. VII. 1991, P.S. \& R.V., 1 ind. - Egina, Ag. Marina, dry grassy meadow, 22. VI. 1986, P.S., abundant.

Anthaxia praeclara Mannerheim, 1837 - Kassandra, Haniotis, on flowers around the church 8. VI. 1985, P.S., abundant.

\section{Subfamily Chalcophorinae}

Chalcophora detrita Klug, 1829 - Thessaly, Skiathos, Karavotsakisma-Madraki, pine forest, 6. VII. 1991, P.S. \& R.V., several ind. - Kassandra, Haniotis, 11. VI. 1987, P.S., abundant. - Peloponnesos, Olympia, pine forest, 9.10.VI.1987, P.S. and 1. VII. 1989, R.V., several ind. on both occasions.

\section{Subfamily Chrysobothrinae}

Chrysobothris affinis (Fabricius, 1794) - Zagoria, Kipi, on oak trunks, 24. VII. 1991, P.S. \& R. V., several ind. Thessaly, Skiathos, 25. VI. 1991, P.S. \& R.V., 1 ind. Delloi, on Populus, 17. VI. 1986, P.S., 1 ind. Polyphagous on deciduous trees.

\section{Subfamily Agrilinae}

Coraebus rubi (Linnaeus, 1767) - Thessaly, Skiathos, 25. VI. 1991, P.S. \& R.V., 1 ind.

Agrilus cyanescens (Ratzeburg, 1837) - Skiathos, 24. VI. 1991, P.S. \& R.V., 1 ind. - On trunks and branches of Lonicera species.

Agrilus viridis (Linnaeus, 1758) - Skiathos, Megaloammos, 7. VII. 1991, P.S. \& R.V., 1 ind. - Polyphagous on deciduous trees and bushes.

\section{Cerambycidae, subfamily Prioninae}

Megopis scabricornis (Scopoli, 1763) - Mt. Pilion, Zagora, $500 \mathrm{~m}, 2$. VIII. 1991, R.V., remnants of 1 ind. on the road.

Prionus coriarius (Linnaeus, 1758) - Thessaly, Skiathos, 25.VI., 
3. VII. 1991, P.S. \& R.V., several ind. on light and on trees. Polyphagous on both deciduous and coniferous trees (von Demelt 1982).

\section{Subfamily Spondylinae}

Arhopalus ferus (Mulsant, 1839) (= tristis F.). - Skiathos, 31. VII. 1991, P.S. \& R.V., 1 ind. - Kassandra, Haniotis, 8. VI. 1985, P.S., 1 ind.

Saphanus ganglbaueri Brancsick, 1886 - Mt. Pilion, Hania, $1500 \mathrm{~m}$, sugar bait trap, 23. VII. 1991, P.S. \& R.V., 2 ind.

\section{Subfamily Cerambycinae}

Hesperophanes sericeus (Fabricius, 1787) - Thessaly, Mt. Pilion, Zagora, 500 m, 2. VIII. 1991, R.V., 1 ind.

Trichoferus griseus (Fabricius, 1792) - Thessaly, Mt. Pilion, Makrinitsa, $800 \mathrm{~m}$, ravine with Platanus and other deciduous trees, light trap, 31. VII. 1991, R.V., 1 ind.

Stromatium fulvum (Villiers, 1789) - Thessaly, Mt. Pilion, Makrinitsa, 800 m, 31. VII. 1991, R.V., 2 ind. - On Quercus and Acer (Berger 1987).

Cerambyx cerdo Linnaeus, 1758 - Delfoi, on fruit trees near the village, 17. VI. 1986, several ind. in the evening. - Arahova, 24. VI. 1989, R.V., several ind. on thistles. Peloponnesos, Andritsena, oak forest, 30. VI. 1989, R.V., several ind.

Cerambyx scopolii Füesslins, 1775 - Skiathos, Koukonaris, 6. VII. 1991, P.S. \& R.V., 1 ind. - Zagoria, Monodendri Balcony, 29. VI. 1991, P.S. \& R.V., 5 ind. - Peloponnesos, Andritsena, oak forest, 30. VI. 1989, R.V., several ind. On deciduous trees.

Purpuricenus budensis (Göetz, 1783) - Kassandra, Haniotis, 8. VI., 11. VI. 1985, P.S., 4 ind. - Peloponnesos, Kalavrita, on thistle flowers, 20. VI. 1986, P.S., abundant. - Delfoi, 16. VI. 1986, P. S., 1 ind - Thessaly, Makrihori, on thistle flowers, 13. VI. 1985, P. S., abundant. - Zagoria, Monodendri Balcony, 29. VI: 1991, P.S. \& R.V., several ind.

P. kaehleri (Linnaeus, 1758) - Skiathos, Koukonaris, 6. VII. 1991, P.S. \& R.V., 1 ind. - Recorded on Quercus coccifera from Zachlorou (von Demelt 1967).
P. desfontainei (Fabricius, 1792) - Arahova-Delfoi, dry roadside meadows, on thistles, 24. VI. 1989, R.V., several ind.

Stenopterus rufus (Linnaeus, 1767) -Skiathos, Aselinos, 5. VII. 1991, P.S. \& R.V., 1 ind. - Kassandra, Haniotis, 7. VI., 8. VI. 1985, P. S., 5 ind. - Kalambaka, Meteora, 13. VI. 1988, P.S., 1 ind.

Hylotrupes bajulus (Linnaeus, 1758) - Skiathos, Megaloammos, light trap, 25. VI., 7. VII. 1991, P.S. \& R.V., 3 ind. - Kamena Vourla, 14. VI. 1988, P.S., 1 ind. — Delfoi, 16. VI. 1985, P.S., 1 ind.

Ropalopus clavipes (Fabricius, 1775) - Skiathos, Megaloammos, 25. VI., 3. VII. 1991, P.S. \& R.V., 2 ind. - Kamena Vourla, 14. VI. 1988, P.S., 1 ind.

Leioderus kollari Redtenbacher, 1849 - Delfoi, 16. VI. 1986, P.S., 1 ind

Plagionotus scalaris (Brullé, 1832) - Kalambaka, Meteora, 13. VI. 1988, P.S., 1 ind-- Peloponnesos, Kalavrita, 20. VI. 1986, P.S., 1 ind.

Echinocerus floralis (Pallas, 1733) - Epirus, Zagoria, Kipi, Kokouli bridge, 27. VI., 24. VII. 1991, P.S. \& R.V., 2 ind.-Peloponnesos, Kalavrita, 20. VI. 1986, P.S., 1 ind. - Kassandra, Haniotis, 11.VI. 1985, P.S., 1 ind.

Xylotrechus arvicola (Olivier, 1795) - Skiathos, Megaloammos, 3.7.1991, P.S. \& R.V., 2 ind. in gardens. Zakynthos, Porto Zorro, 0-30 m, 8. VI. 1987, P.S., 1 ind.

Chlorophorus sartor (Müller, 1766) - Skiathos, Megaloammos, 7. VII. 1991, P.S. \& R.V. 1 ind. - Kassandra, Haniotis, 8. VI., 11. VI., 18. VI. 1985, P.S., 5 ind.

C. figuratus (Scopoli, 1763) - Kalambaka, Meteora, 13. VI. 1988, P.S., 1 ind.

C. varius (Müller, 1766) - Skiathos, near town, on meadows and roadside flowers, 3. VII. 1991, P.S. \& R.V, abundant. - Skiathos, Aselinos, on roadside flowers, 5. VII. 1991, P.S. \& R.V., abundant. - Egina, Ag. Marina, 13. VI., 22. VI. 1986, P.S., 2 ind. - Kassandra, Haniotis, 8. VI. 1985 , P.S., 1 ind. 
Clytus rhamni Germar, 1817 - Skiathos, 25. VI. 1991, P.S. \& R.V., 1 ind. - Kassandra, Haniotis, 8.VI. 1985, P.S., 2 ind.

Anastrangalia sanguinolenta (Linnaeus, 1761) - Epirus, Katara Pass, 1650 m, 1. VII. 1991, R.V.\& P.S., 1 ind.

Stictoleptura cordigera (Fuiesslins, 1775) - Skiathos, Megaloammos, 25. VI., 3. VII. 1991, P.S. \& R.V., 4 ind. - Skiathos, Aselinos, 5. VII. 1991, P.S. \& R.V., 1 ind. Peloponnesos, Kalavrita, 20. VI. 1986, P.S., 1 ind.

Vadonia moesiaca (Daniel, 1891) - Arahova, dry meadows above the village, 24. VI. 1989, R.V., several ind. Zagoria, Tymphi, Goura, $2000 \mathrm{~m}$, on flowers, 28. VI., 25. VII. 1991, P.S. \& R.V., abundant.

Pseudovadonia livida (Fabricius, 1776) - Kassandra, Haniotis, 8. VI. 1985, P.S., 4 ind.

Rutpela maculata (Poda, 1761) - Skiathos, Megaloammos, 25. VI., 3. VII. 1991, P.S. \& R.V., 3 ind. - Kalambaka, Meteora, 13. VI. 1988, P.S., 2 ind. - Peloponnesos, Kalavrita, 20. VI. 1986, P.S., 1 ind.

Stenurella bifasciata (Müller, 1776) - Skiathos, Megaloammos, on flowers, 25. VI., 3. VII. 1991, P.S. \& R.V., abundant. - Skiathos, Aselinos, 5. VII. 1991, P.S. \& R.V., several ind.

S. septempunctata (Fabricius, 1792) - Zagoria, Monodendri Balcony, 29. VI. 1991, P.S. \& R.V., 1 ind. Zagoria, Kipi, 24. VII. 1991, P.S. \& R.V., 1 ind. Kalambaka, Meteora, 13. VI. 1988, P.S., 3 ind.

\section{Subfamily Lamiinae}

Niphona pictinicornis Mulsant, 1839 - Skiathos, 25. VI., 4. VII., 4. VIII. 1991, P.S. \& R.V., 3 ind.

Dorcatypus tristis (Linnaeus, 1767) - Kastoria, 1. VI. 1988, P.S., 1 ind.

Morimus asper (Sulzer, 1776) - Zagoria, Papingo, river valley with deciduous forest, 26. VII, 1991, P.S. \& R.V., 1 ind. Neodorcadion bilineatum (Germar, 1824) - Kassandra, Haniotis, 8. VI. 1985, P.S., 1 ind. - Kalambaka, 12. VI. 1988 , P.S., 1 ind.
Acanthoderes clavipes (Schrank, 1781) - Skiathos, Megaloammos, 24. VI., 7. VII 1991, P:S. \& R.V., 2 ind.

Acanthocinus griseus novaki Tippermann, 1952 -Zagoria, Tsepelovo, pine forest, on light, 24. VII. 1991, P.S. \& R.V., 1 ind.

Agapanthia cynarae (Germar, 1817) - Skiathos, roadside meadows, on flowers, 25. VI. 1991, P.S. \& R.V., several ind. - Peloponnesos, Kalavrita, on thistle flowers, 20. VI. 1986, P.S., abundant. — Kassandra, Haniotis, 8. VI: 1985 , P.S., 1 ind. - Thessaly, Ossa, Tembi, 9. VI. 1985, P.S., abundant.

Saperda scalaris (Linnaeus, 1758) - Near Pades at the foot of Mt. Smolikas, 29.VI. 1991, P.S. \& R.V., 1 ind.

Oberea taygetana Pic, 1904 - Thessaly, Mt. Pilion, Makrinitsa, meadow with Euphorbia characias subsp. wulfenii (Koch) A.R.Sm, above the village, $800 \mathrm{~m}, 2$. VII. 1991 , P.S. \& R.V., 1 ind.

Mallosia graeca Sturm, 1843 - Peloponnesos, Vitina, meadow, 100-1100 m, 11.-12. VI. 1987, P.S., abundant.

Acknowledgements. A grant from the Ministry of Education for cultural cooperation between Finland and Greece is gratefully acknowledged.

\section{References}

Berger, P. 1987: Un voyage entomologique en Crète (713.VI.1981). - Biocosme Mesogeen. 4:233-248.

Demelt, C. von, 1967: Beitrag zur Kenntnis der Cerambycidenfauna Griechenlands (Col.). _ Entomol. Zeitschr. 77:57-66.

Demelt, C. von, 1982: Nachtrag zur Kenntnis der Cerambycidenfauna Griechenlands (ohne Inseln). Entomol. Zeitschr. 92:240-242.

Lodos, N. \& Tezcan, S. 1990: General aspect of buprestid beetle fauna of Turkey (Coleoptera: Buprestidae). 5th Intern. Congr. Zoogeogr. \& Ecol. Greece \& Adjacent Areas, Iraklion, Crete, Hellenic Zool. Soc., (Abstracts), p. 13.

Markalas, S. 1991: Rinden- und holzbohrende Insektenarten in Kiefern (Pinus halepensis, P. brutia und P. nigra) nach Waldbränden in Griechenland. - Anz. Schädlingskde. Pflanzenschutz Umweltschutz 64:72-75.

Sama, G. 1988: Coleoptera Cerambycidae. Catalogo topografico e sinonimico. - Fauna d'Italia, Edizioni Calderini, Bologna, 216 pp. 